\title{
Long-term respiratory complications of OA-TOF: The need for better data and a more specialised multidisciplinary approach
}

\author{
Paul D Losty ${ }^{1}$ and Rebecca Thursfield ${ }^{1}$ \\ ${ }^{1}$ Alder Hey Children's NHS Foundation Trust
}

September 28, 2020

\begin{abstract}
Editorial: Long-term respiratory complications of OA-TOF: The need for better data and a more specialised multidisciplinary approach
\end{abstract}

\section{Editorial}

Long-term respiratory complications of OA-TOF: The need for better data and a more specialised multidisciplinary approach

Paul D. Losty ${ }^{1}$, Rebecca Thursfield ${ }^{2}$

1. Department Of Paediatric Surgery, Alder Hey Children's Hospital NHS Foundation Trust, School Of Health And Life Science, University of Liverpool, UK

2. Department Of Paediatric Respiratory Medicine, Alder Hey Children's Hospital NHS Foundation Trust, Liverpool, UK

Tracheoesophageal fistula (TOF) are caused by the failed fusion of the tracheoesophageal ridges around the fourth week of embryonic development. Closely linked with maldevelopment of the foregut it is commonly associated with oesophageal atresia (OA). Congenital oesophageal atresia, with or without TOF, occurs in approximately 1 in 3500 live births. The lesion may be suspected antenatally in a minority of cases $(25 \%)$ associated with polyhydramnios though most index cases are diagnosed in the new-born period. Many babies present within the first days of life with inability to tolerate feeds and diagnosis is linked to the inability to secure passage of a nasogastric tube into the infant's stomach. Surgical repair is promptly scheduled after diagnosis with closure of the fistula tract and anastomosis of the oesophagus. Newborns with a " long gap " in the separated oesophageal segments cannot readily have primary repair due to the risk of " anastomotic breakdown " if the oesophagus is brought together by the surgeon under high suture tension. Here, ' delayed primary repair " with a period of gastrostomy feeding and "sham" oral feeds with a cervical oesophagostomy ensures the child can acquire key oral motor skills for later definitive repair. If delayed repair of the oesophagus is not feasible after some 8 weeks, oesophageal replacement should be undertaken with varied options available including gastric transposition, a colon graft, or jejunal interposition .

No matter what surgical technique(s) are deployed even with primary repair of the oesophagus where anatomical correction is achieved, significant functional abnormality(s) remain thereafter for patients. There are a multitude of factors to consider which are herein discussed in the paper by Koumbourlis, Belessis et al in this issue of Pediatric Pulmonology. These functional abnormalities can cause life-long morbidity and impact patients and families in many different ways. Some morbidities are very specific, such as food bolus obstruction at the site of the oesophageal anastomosis. Others lead to much more vague troublesome symptoms notably chesty cough, breathlessness, vomiting, poor appetite, epigastric pain, chest infections and 
failure to thrive. If these patients are seen by health care professionals without adequate experience of TOF - OA, symptoms here may not be recognised such that accurate diagnosis and planned management can be delayed. The problems are often multifactorial and for optimal health care these children should ideally be managed in specialist clinics staffed by multidisciplinary teams including, paediatric surgeons, respiratory physicians, dieticians, physiotherapists, speech and language therapists (SALT), ENT surgeons, medical gastroenterology and psychologists. Access to such key specialists appears to be highly variable amongst centres; one study found that two thirds of patients developed respiratory symptoms and most were seen by appropriate specialists, whilst another single centre paper highlighted that only half of all patients with respiratory or gastrointestinal problems were seen by the appropriate specialist. These two articles clearly highlight wide clinical practice variation amongst such centres and a lack of consistency in MDT care. Delay in accurate diagnosis of respiratory sequale of OA-TOF can lead to poor quality of life (QoL), development of oral feeding aversion and marked failure to thrive with poor caloric intake and increased energy expenditure linked with tachypnoea and bronchiectasis through recurrent infections.

For parents of children with TOF-OA, medical problems are combined with the task of managing day to day issues faced in "normal" life. There is increased parental anxiety at milestone points such as weaning to infant diets and starting school, added to numerous hospital clinic visits and other surgical operations for some of our more vulnerable patients. For a birth defect that is characterised with such morbidity surprisingly few robust multicentre data exist including care pathway guidelines. As Koumbourlis, Belessis et al discuss, most current data emerge from single centre experience and here we agree there is a crucial need for larger scale collaborative studies. This manuscript is therefore timely in Pediatric Pulmonology as a 'work in progress' consensus on the utility of diagnostic studies and therapeutics addressing respiratory complications of OA-TOF patients.

The article is a good starting point aimed at developing better outcome data for OA-TOF patients and the authors should be congratulated for this work. The GRADE methodology was rejected by the study authors due to the lack of good quality evidence currently available. Instead, the RAND appropriateness method (RAM) was adopted by authors. The resultant consensus recommendations are thorough and cover all aspects of respiratory management of patients with OA-TOF. The study authors make 37 recommendations on various diagnostic techniques and 25 key points on respiratory interventions including management of tracheobronchomalacia, antibiotic treatment, chest physiotherapy and therapy of gastro-oesophageal reflux disease. The authors also describe the need for multidisciplinary (MDT) follow-up. After care follow-up of every child with OA-TOF in a specialist centre is essential ideally with a multidisciplinary team to better detect long term morbidity(s) with prompt better treatments and outcomes.

Advances in neonatal surgery with better intensive care over the past decade(s) are such that excellent survival $(>97 \%)$ is the norm expected. Outcomes in the modern era of care must therefore focus on QoL and long-term health. In order to set better outcomes for OA-TOF children tools such as core outcomes measures (CoS) are required. Such metrics are currently lacking and collaborative development with health care professionals and parent support groups notably TOFS UK and EATS is important .

Large scale multi-centre collaborative national and international data should be developed. Longitudinal follow up data and registries would allow for better understanding of the nature and extent of the problems faced by OA -OF patients and families and more tailored evidence based management plans.

This current manuscript by Koumbourlis, Belessis et al in Pediatric Pulmonology is a step forward in the right direction towards consensus based management of respiratory health in OA-TOF children. We now need to work together as health care professionals to establish core outcomes, collate national and international data (with registries) and ensure these special children receive the best care possible for a long and healthy life.

1. P, Losty. Esophageal Atresia and Tracheo-Esophageal fistula . [book auth.] Flake A, Rintala R, Hutson J, iwai N Losty P. Rickhams neonatal Surgery. London : Springer Publishers, 2018, 23, pp. 541-563.

2. Porcaro F, Valfre L, Rotondi Aufiero L, Angelis P, Villani A, Bagolan P, Bottero S and Cutrera R. Respiratory problems in children with esophageal atresia and tracheoesophageal fistula. 1, 
2017, ItalianJournal of Paediatrics, Vol. 43, p. 77.

3. DeBoer E, Prager J, Ruiz A, Jenson E, Deterding R, Friedlander JA, Sodon J. Multidisciplinary care of children with repaired esophageal atresia and tracheoesophageal fistula. 6, 2016,Pediatric Pulmonology, Vol. 51, pp. 576-81.

4. Koumbourlis A, Belessis Y, Cataletto M, Cutera R, DeBoer E, Kazachkov M, Laberge S, popler J, Porcaro F, Kovesi T. Care Recommendations for the Respiratory Complications of Esophageal Atresia-Tracheoesophageal Fistula: The International Network of Esophageal Atresia, Respiratory Complications Working Group. 2020,Pediatric Pulmonology .

5. TOFS. [Online] https://www.tofs.org.uk/home.aspx.

6. EAT. Federation of Esophageal atresia and trache-eosophageal fistula support groups. [Online] https://www.we-are-eat.org/. 